\title{
P-Grid: A Self-organizing Access Structure for P2P Information Systems
}

\author{
Karl Aberer \\ Distributed Information Systems Laboratory \\ Department of Communication Systems \\ Swiss Federal Institute of Technology (EPFL) \\ 1015 Lausanne, Switzerland \\ karl.aberer@epfl.ch
}

\begin{abstract}
Peer-To-Peer systems are driving a major paradigm shift in the era of genuinely distributed computing. Gnutella is a good example of a Peer-To-Peer success story: a rather simple software enables Internet users to freely exchange files, such as MP3 music files. But it shows up also some of the limitations of current P2P information systems with respect to their ability to manage data efficiently. In this paper we introduce P-Grid, a scalable access structure that is specifically designed for Peer-To-Peer information systems. P-Grids are constructed and maintained by using randomized algorithms strictly based on local interactions, provide reliable data access even with unreliable peers, and scale gracefully both in storage and communication cost.
\end{abstract}

Keywords. Peer-To-Peer computing, Distributed Indexing, Distributed Databases, Randomized Algorithms.

\section{Introduction}

Peer-To-Peer (PQP) systems are driving a major paradigm shift in the era of genuinely distributed computing [2]. Major industrial players believe "P2P reflects society better than other types of computer architectures. It is similar to when in the 1980's the PC gave us a better reflection of the user" (www.infoworld.com).

In a $\mathrm{P} 2 \mathrm{P}$ infrastructure, the traditional distinction between clients and backend (or middle tier application) servers is simply disappearing. Every node of the system plays the role of a client and a server. The node pays its participation in the global exchange community by providing access to its computing resources. Gnutella is a good example of a P2P success story: a rather simple software enables Internet users to freely exchange files, such as MP3 music files.

In the current P2P file-sharing systems, like Gnutella [1], no indexing mechanisms are supported. Search requests are broadcasted over the network and each node receiving a search request scans its local database (i.e. its file system) for possible answers. This approach is extremely costly in terms of communication and leads to high search costs and response times. For supporting efficient search, however, appropriate access structures are prerequisite. 
Access structures in distributed information systems have been addressed in the area of distributed and parallel databases. Different approaches to data access in distributed environments have been taken. We mention some of the principal approaches.

- The distribution of one copy of a search tree over multiple, distributed nodes is a technique that has been investigated in [6]. The same authors have shown that, under certain assumptions, with that approach balanced search trees do not exist [7].

- The replication of the complete search structure is an approach that underlies the $R P^{*}$-Trees proposed in [8]. In [10] a mechanisms is proposed that leads eventually to the replication of the search structures.

- Scalable replication of a search tree (more precisely B-Tree) is proposed in [5] (dB-Tree) and [11] (Fat-BTree). With scalable replication each node stores a single leaf node of the search tree, the root node of the search tree is replicated to every node, and the intermediate nodes are replicated such that each node maintains a path from the root to its own leaf.

- No search structures: in these approaches operation messages are broadcasted to all participating nodes. E.g. with $R P_{N}^{*}$ [8] the data is range partitioned as in B-Trees but no index exists and a multicast mechanism is used. In current P2P file sharing systems, like Gnutella, the P2P network is used to propogate search requests to all reachable peers.

Many of these approaches assume a reliable execution environment, require some centralized services, are designed for a fairly small numbers of nodes (hundreds) and focus on deterministic execution guarantees. In this paper we would like to take a different approach and address the question of how an access structure can be built by a community of a very large number of unreliable peers without any central authority, that can provide still a certain level of reliability of search and scales well in the number of peers, both in storage and communication cost. In order to obtain scalability we use the approach of scalable replication of tree structures, as proposed in [5][11]. To construct these search structures and perform searches and updates we use randomized algorithms, that are based exclusively on local interactions among peers. The idea is that by randomly meeting among each other the peers successively partition the search space and retain enough information in order to be able to contact other peers for efficiently answering future search requests. The resulting distributed access structure we call P-Grid (Peer-Grid). As this paper addresses the prinicipal feasibility of such an approach we make the simplifying assumption that the data distribution is not skewed. Thus the use of binary search trees over a totally order domain of keys is sufficient (as e.g. also used in [6]). Similarly, for the analysis and simulation of PGrids, we make uniformity assumptions on the peer behavior. Though definitely in a next step skewed data distributions and extended methods for balancing the search trees, as well known from B-Tree structures, are required, even the basic methods in this paper can be beneficial to improve the efficiency of current file sharing applications. The main characteristics of P-Grids are that 
- they are completely decentralized, there exists no central infrastructure, all peers can serve as entry point to the network and all interactions are strictly local.

- they use randomized algorithms for constructing the access structure, updating the data and performing search; probabilistic estimates can be given for the success of search requests, and search is robust against failures of nodes.

- they scale gracefully in the total number of nodes and the total number of data items present in the network, equally for all nodes, both, with respect to storage and communication cost.

In the next section we introduce our system model and the structure of P-Grids. In Section 3 we describe the distributed, randomized algorithm that is used to construct P-Grids. In Section 4 we give some analysis on basic properties of P-Grids. Section 5 contains extensive simulation results, that demonstrate the feasibility of the P-Grid algorithms and exhibit important scalability properties of P-Grids. Section 6 indicates directions for future work.

\section{System Model and Access Structure}

We assume that a community of peers $P$ is given that can be addressed using a unique address addr $: P \rightarrow A D D R$. For an address $r \in A D D R$ we define $\operatorname{peer}(r)=a$ iff $a d d r(a)=r$ for $a \in P$. The peers are online with a probability online $: P \rightarrow[0,1]$. Peers that are online can be reached reliably through their addressusing the underlying communication infrastructure.

Every peer stores information items from a set $D I$ that are characterized by an index term from a set $K$. The set of index terms is totally ordered, such that a search tree can be constructed in the usual way. In the following, we assume that the index terms are binary strings, built from 0's and 1's and that a key $k=p_{1} \ldots p_{n}, p_{i} \in\{0,1\}$ corresponds to a value $\operatorname{val}(k)=\sum_{i=1}^{n} 2^{-i p_{i}}$ and an interval $I(k)=\left[\operatorname{val}(k), \operatorname{val}(k)+2^{-n}[\subseteq[0,1[\right.$.

Now we define the access structure. The goal is to construct an access structure, such that

- The search space is partitioned into intervals of the form $I(k), k \in K$. Every peer takes over responsibitity for one interval $I(k)$. As each key corresponds to a path in the binary search tree we will also say that the peer is responsible for the path $k$.

- Taking over responsibility for an interval $I(k)$ means, that a peer should provide the addresses of all peers that have an information item with a key value $k_{\text {query }}$ that belongs to $I(k)$, i.e. $\operatorname{val}\left(k_{\text {query }}\right) \in I(k)$.

- For each prefix $k_{l}$ of $k$ of length $l, l=1, \ldots$, length $(k)$ a peer $a$ maintains references to other peers, that have the same prefix of length $l$, but a different value at position $l+1$, for the key they are responsible for. We will call these references to other peers, $a$ 's references at level $l+1$. These references are used to route search requests for keys with prefix $k_{l}$, but a continuation that does not match the own key, to other peers. 
- A search can start at each peer.

Before giving the formal definition of the access structure, we give in Fig. 1 a simple example. The different levels relate to the different levels of the binary search tree. The intervals relate to the nodes of the search tree. We indicate the key values that correspond to the intervals (i.e. 0 and 1 at level 1, 00, 01, 10, and 11 at level 2). At the lowest level we entered have 6 peers, indicated by black circles, into the leaf nodes corresponding to the keys they are responsible for. Multiple peers can be responsible for the same key. We will call these later also replicas. We also entered the agents into all intervals on the path from the root to their leaf node. At the leaf nodes we show the pointers to specific data items whose key is a prefix of the key of the peer.

At level zero every peer is associated with the whole interval, in other words, it stores a root node of the search tree. At level 1 every peer is associated with exactly one of the two intervals. At level 2 every peer is associated with exactly one interval. The connectors from one level to the next are the references that $\mathrm{s}$ peer maintains to cover the other side of the search tree. For example, at level 0 peer 1 has a reference to peer 3 , and at level 1 peer 1 has a reference to peer 2 .

When a search request is issued it is routed over the responsible peers. There are two possiblities, either at the next level the peer itself is responsible, then it can further process the request itself, or, the request needs to be forwarded to another peer. For illustration we have included into Fig. 1 the processing of two queries. In the first example the query 00 is submitted to peer 1 . As peer 1 is reponsible for 00 it can process the complete query. In the second example the query 10 is submitted to peer 6 . Using its reference at level 0 it contacts peer 5, which in turn contacts at level 1 peer 4 , who is responsible for the key corresponding to the query.

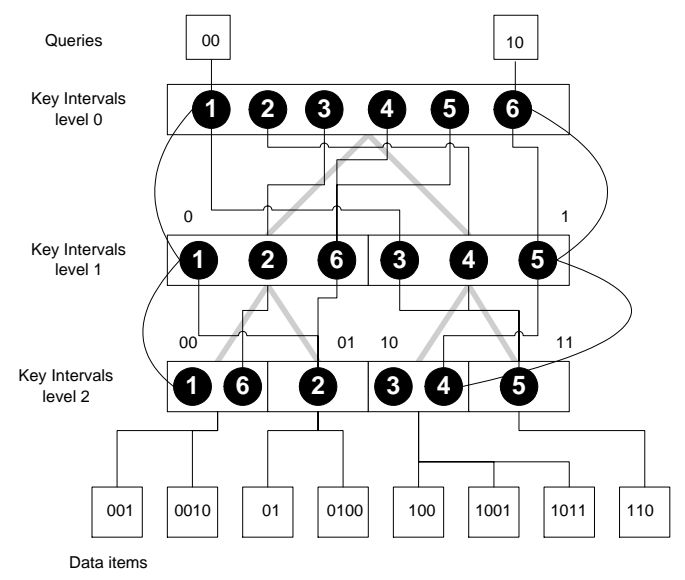

Fig. 1. Example P-Grid 
We define now formally the data structure for peers that allows to represent the P-Grid. Every peer $a \in P$ maintains a sequence

$$
\left(p_{1}, R_{1}\right)\left(p_{2}, R_{2}\right) \ldots\left(p_{n}, R_{n}\right),
$$

where $p_{i} \in\{0,1\}$ and $R_{i} \subset A D D R$. We define path $(a)=p_{1} \ldots p_{n}$, prefix $(i, a)=$ $p_{1} \ldots p_{i}$ for $0 \leq i \leq n$ and $\operatorname{refs}(i, a)=R_{i}$. In addition the number of references in $R_{i}$ will be limited by a value refmax. The sets $R_{i}, 1 \leq i \leq n$ are references to other peers and satisfy the following property:

$$
r \in \operatorname{ref} s(i, a): \operatorname{prefix}(i, \operatorname{peer}(r))=\operatorname{prefix}(i-1, a) p_{i}^{-}
$$

where $\operatorname{path}(a)=p_{1} \ldots p_{n}$ and $p^{-}$is defined as $p^{-}=(p+1) M O D 2$. In addition, each peer maintains a set of references $D \subset A D D R \times K$ to the peers that store data items indexed by keywords $k$ for which path $(a)$ is a prefix. In other words, at the leaf level the peer knows at which peers data items corresponding to the search keys that it is responsible for, can be found.

This gives rise to a straightforward depth first search algorithm shown in Fig. 2. Given a P-Grid, a query $p$ can be issued to each peer $a$ through a call query $(a, p, 0)$.

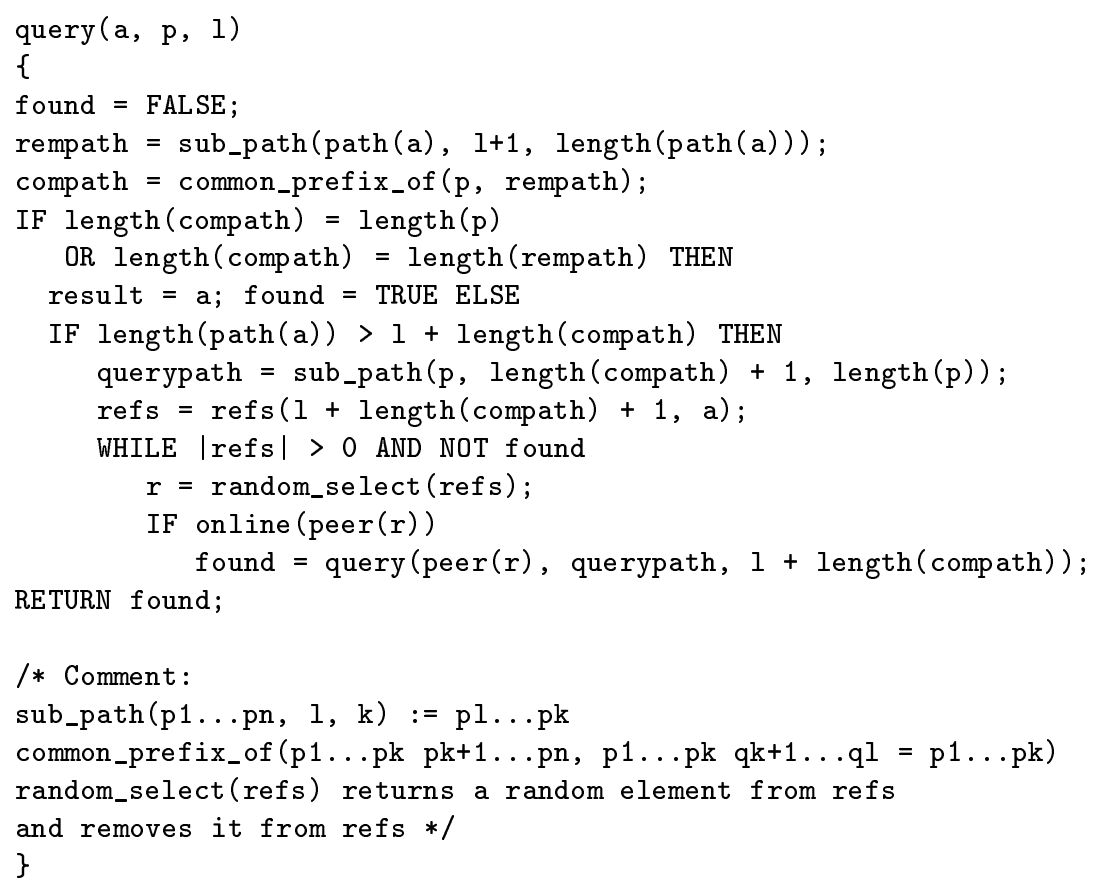

Fig. 2. P-Grid search algorithm 


\section{P-Grid Construction}

Having introduced the access structure and the search algorithm, the main question is now, of how a P-Grid can be constructed. As there exists no global control this has to be done by using exclusively local interactions. They idea is that whenever peers meet, they use the opportunity to create a refinement of the access structure. We do not care at this point why peers meet. They may meet randomly, because they are involved in other operations, or because they systematically want to build the access structure. But assuming that by some mechanisms they meet frequently, the procedure works as follows.

Initially, all peers are responsible for the whole search space, i.e. all search keys. At that stage, when two peers meet initially, they decide to split the search space into two parts and take over responsibility for one half each. They also store the reference to the other peer in order to cover the other part of the search space. The same happens whenever two peers meet, that are responsible for the same key at the same level.

However, as soon as the P-Grid develops, also other cases occur. Namely, peers will meet where their keys share a common prefix or where their keys are in a prefix relationship. In the first case, what the peers can do, is to initiate new exchanges, by forwarding each other to peers they are themselves referencing. In the second case the peer with the shorter key can specialize by extending its key. In order to obtain a balanced P-Grid it will specialize in the opposite way the other peer has already done at that level. The other peer remains unchanged. These considerations give rise to the algorithm in Fig. 3 that two peers $a 1$ and $a 2$ execute when they meet.

A few remarks are in place on this algorithm. A measure to prevent overspecialization of peers is to bound the maximal length of paths that can be constructed to maxl. Simulations show that this results in a more uniform distribution of path lengths among peers and better convergence of the P-Grid. Also such a bound is needed when a certain degree of replication at the lowest grid level is to be achieved. The disadvantage is that some global knowledge is used, namely the maximal path length, which not always might be locally available or easily derivable. In practical applications, one possible indication that a path has reached maxl could be that the number of data items belonging to the key is falling below a certain threshold.

An alternative would be to avoid overspecialization by taking another approach in Case 2 and Case 3, where one path is subpath of the other and the peer with shorter path chooses to specialize differently than the other peer. Here one could shorten the longer path if the difference in length is greater than 1 , such that both resulting paths have the same length. However, this would require additional means to maintain consistency of references as peers give up responsibility for keys by generalizing and could possibly specialize at a later stage differently. This approach also slows down convergence. So we ommitted this possibility here.

The recursive executions of the exchange function by using the locally available references (Case 4) have an important influence on the performance of the 


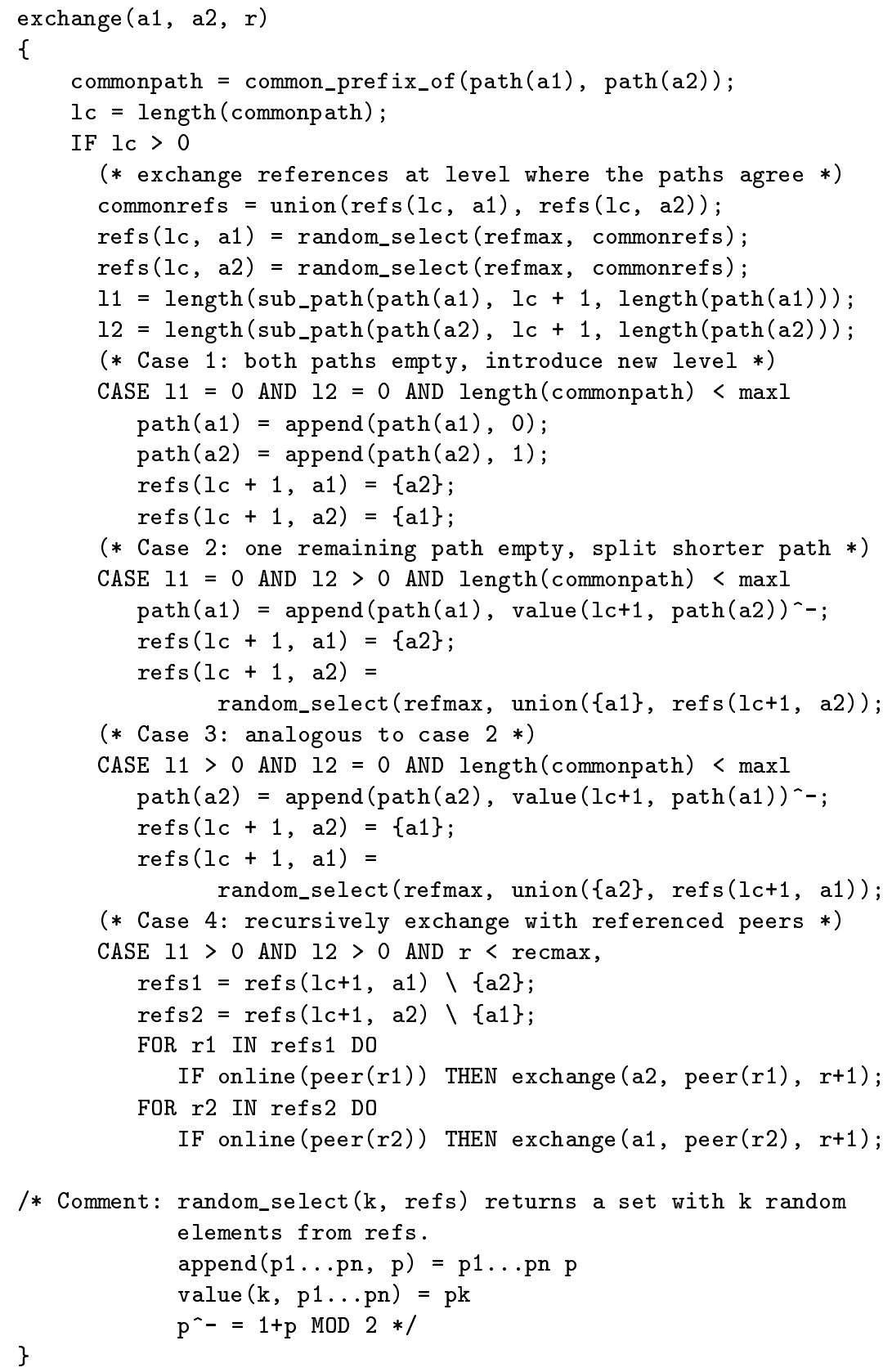

Fig. 3. P-Grid construction algorithm 
method. These executions are more promising of ending up in a successful specialization as they are already targetted to a more specific set of candidates. On the other hand the recursive executions might lead to a quick overspecialization of the P-Grid for subregions of the search tree. Therefore, we bound the recursion depth up to which the exchange function is called by the value recmax. This value has a very strong influence on the global performance of the algorithm, as we will see later.

So far, we have only considered the construction process of the access structure itself. At the leaf level the peers need also to know the data items, respectively the peers storing those data items, that correspond to their responsibility. As many peers can be responsible for the same key the general problem is of how to find all those peers in case of an update. Different strategies are possible:

- Randomly performing depth first searches for peers responsible for the key multiple times and propagating the update to them

- Performing breadth first searches for peers responsible for the key once and propagating the update to them

- Creating a list of buddies for each peer, i.e. other peers that share the same key, and propagate the update to all buddies.

We will not give the detailed algorithms here as they are quite obvious. But in Section 5 we will identify by using simulations, which is the most efficient method.

\section{Analysis of Search Performance}

We want to analyze the question of how probable it is to find a peer that is responsible for a specific search key starting the search at an arbitrary peer. This analysis allows to give rough estimates on the sizing of the P-Grid parameters that are required to achieve a desired search reliability in a given setting. We perform the analysis for an idealized situation, where for all peers the parameters of the P-Grid, like keylength and number of peers responsible for the same key, are uniformly distributed. Though such a distribution will not occur in practice it gives a good estimation the quantitative nature of a P-Grid.

The following parameters determine the problem. The number of peers $N$ and the number of data objects each peer can store $d_{p e e r}$ determine the total number of data objects that can be stored in the network as $d_{\text {global }}=N * d_{\text {peer }}$. The size of a reference $r$ and the amount of space each peer is willing to make available for indexing purposes $s_{\text {peer }}$ determines the possible number of references that can be stored at each peer $i_{\text {peer }}=\frac{s_{\text {peer }}}{r}$.

Now we determine the number of entries required for a certain grid organisation. The length of a key required to differentiate data items located at different peers is given by

$$
k \geq \log _{2} \frac{d_{\text {global }}}{i_{\text {leaf }}}
$$


where $i_{\text {leaf }}$ is the number of references to data items each peer stores at the leaf level.

Then the total number of index entries stored at a peer is given by $i_{\text {leaf }}+$ $k *$ refmax , where refmax is the multiplicity of references used to build the grid structure. Thus we obtain the constraint $i_{\text {leaf }}+k *$ refmax $\leq i_{\text {peer }}$ which determines the value of $i_{\text {leaf }}$. In order to allow at the lowest level of the grid the support for refmax alternative peers, references to data items need to be replicated with a factor of refmax. This is only possible if

$$
\frac{d_{\text {global }}}{i_{\text {leaf }}} * \text { refmax } \leq N
$$

i.e. there must be sufficiently high numbers of peers available, such that each interval at the lowest grid level is supported by at least re fmax peers.

Given a constant probability $p$ that a peer is online we are now interested in the question what is the probability of performing a successful search for a peer that is responsible for the query key. In the worst case at each level of the grid a new peer needs to be contacted, that is selected out of the available references. At each level of the grid, the probability of reaching a peer at the next level is $1-(1-p)^{r e f \max }$, i.e. one minus the probability that all refmax referenced peers are offline. Since the search tree is of depth $k$, then the probability of performing a successful search for a key is

$$
\left(1-(1-p)^{\text {refmax }}\right)^{k}
$$

We give now an example, to illustrate what a P-Grid would cost in terms of space for a practical setting.

Example. Let us consider the setting P2P file sharing as it currently is found with Gnutella. We use some rough estimates of the actual parameters that are observed for this application. Assume that $d_{\text {global }}=10^{7}$ data objects (files) exist, that a reference costs at most $r=10$ Bytes of storage (the path plus the IP address) and that every peer is willing to provide $s_{\text {peer }}=10^{5}$ Bytes of storage for indexing (which is in fact far less than the size of an average media file). Furthermore, we assume that peers are online with probability $30 \%$.

Let us now analyze how large a community for supporting the $10^{7}$ files must be in order to ensure that search requests for files are answered with a probability of $99 \%$. Each peer can store at most $i_{\text {peer }}=10^{4}$ references. If we "guess" a value of $i_{\text {leaf }}=10^{4}-200$, we see that inequality (1) is satisfied for a value of $k=10$. For a value of refmax $=20$ then, according to (3), the probability of sucessfully finding a peer for a given key is larger than $99 \%$. The storage required is due to our good initial guess exactly $s_{\text {peer }}=10^{5}$. The number of peers required to support this grid has to be according to inequality (2) larger than 20409. This is a very reasonable number compared to the size of the actual Gnutella user community. 


\section{Simulation}

For performing simulations we have implemented the algorithm for constructing P-Grids using the mathematical computing package Mathematica. We intend to obtain results on the following questions.

- How many communications in terms of executing the exchange algorithm are required for building a P-Grid ?

- What is the influence of the recursion factor recmax in the exchange algorithm on the efficiency of the P-Grid construction?

- Is the resulting structure reasonably well balanced with respect to distribution of path lengths and number of replicas per path ?

- How reliable can data be found using the P-Grid?

\subsection{Performance of the Construction Method}

In the following simulations we analyze the convergence speed when the PGrid is constructed. The peers meet randomly pairwise and execute the exchange function. We consider a P-Grid as constructed when the average length of the keys that the peers are responsible for reaches a certain threshhold $t$, i.e. $\frac{1}{N} \sum_{a \in P}$ length $($ path $(a))<t$. In the following the path length is bounded by maxl $=6$ and the threshold is $99 \%$ of $\operatorname{maxl}(5.94)$. We count the number of calls to the exchange function $(e)$ to determine the construction cost.

First we investigate the relationship between the number of peers and construction cost. We vary the number of peers from 200 to 1000 . We use a recursion depth recmax of 0 and 2 . The value of refmax was set to 1 , i.e. only one reference to another peer ist stored. This influences the performance in the case where recmax $>0$. The results indicate that a linear relationship exists between the number of peers $(N)$ and the total number of communications $(e)$ needed in building the P-Grid. As a consequence, every peer has to perform on average a (practically) constant number of exchanges to reach its maximal path length independently of the total number of peers involved.

\begin{tabular}{rrrrr}
\hline & recmax $=0$ & \multicolumn{3}{r}{ recmax $=2$} \\
$\mathrm{~N}$ & $e$ & $\frac{e}{N}$ & $e$ & $\frac{e}{N}$ \\
\hline \hline 200 & 15942 & 79.71 & 4937 & 24.68 \\
400 & 27632 & 69.08 & 10383 & 25.95 \\
600 & 43435 & 72.39 & 15228 & 25.38 \\
800 & 59212 & 74.01 & 18580 & 23.22 \\
1000 & 74619 & 74.61 & 25162 & 25.16 \\
\hline
\end{tabular}

The next simulation shows how the choice of a maximal path length maxl influences the number of exchanges $e_{\operatorname{maxl}}$ required. The simulation is done for $N=500$ peers. The results indicate that the number of communications grows exponentially in the path length when no recursion is used. With a recursion bound recmax $=2$ the convergence speeds up substantially. 


\begin{tabular}{rrrrrrr}
\hline maxl & recmax $=0$ & & & recmax $=2$ & \\
$e_{\operatorname{maxl}}$ & $\frac{e_{\operatorname{maxl}}}{N}$ & $\frac{e_{\operatorname{maxl}}}{e_{\max l-1}}$ & $e_{\operatorname{maxl}}$ & $\frac{e_{\operatorname{maxl}}}{N}$ & $\frac{e_{\operatorname{maxl}}}{e_{\operatorname{maxl}}-1}$ \\
\hline \hline 2 & 4893 & 9.78 & & 5590 & 11.18 & \\
3 & 9780 & 19.56 & 1.998 & 7289 & 14.57 & 1.303 \\
4 & 18071 & 36.14 & 1.847 & 8215 & 16.43 & 1.127 \\
5 & 35526 & 71.05 & 1.965 & 13298 & 26.59 & 1.618 \\
6 & 72657 & 145.31 & 2.045 & 17797 & 35.59 & 1.338 \\
7 & 171770 & 343.54 & 2.364 & 27998 & 55.99 & 1.573 \\
\hline
\end{tabular}

The following simulation shows that the recursion depth recmax has substantial influence on the convergence speed. When using recursive calls to the exchange function the probability that a random meeting leads to a successful exchange increases. However, if recursion is not constrained this can lead to negative effects as the peers tend to overspecialize quickly. The result shows that for 500 peers and maximal path length 6 the optimal recursion depth limit is 2 .

\begin{tabular}{rrr}
\hline$r e c m a x$ & $e$ & $\frac{e}{N}$ \\
\hline \hline 0 & 35436 & 70.87 \\
1 & 15377 & 30.75 \\
2 & 12735 & 25.47 \\
3 & 16595 & 33.19 \\
4 & 18956 & 37.91 \\
5 & 22426 & 44.85 \\
6 & 25130 & 50.26 \\
\hline
\end{tabular}

If $\operatorname{ref} \max >1$, i.e. peers maintain more than one reference to other peers at each level, i.e. there exist more possibilities to make recursive calls. Thus if recmax $>0$ this should have an influence on the number of exchanges that are performed when constructing the P-Grid. Note that this additional effort is rewarded by a higher density of the P-Grid. We analyzed this with a simulation with $N=1000$ peers and a recursion depth limit recmax $=2$.

\begin{tabular}{rrr}
\hline$r e f \max$ & $e$ & $\frac{e}{N}$ \\
\hline \hline 1 & 25285 & 25.28 \\
2 & 39209 & 39.20 \\
3 & 72130 & 72.13 \\
4 & 125727 & 125.72 \\
\hline
\end{tabular}

As one can see the number of exchanges grows exponentially, which is undesirable. In fact, this turned out to be a weakness in the algorithm we proposed. However, there exists a simple way to fix this. One limits the number of referenced peers with which exchanges are made throughout recursion. Then the results become very stable as the following simulation shows, where recursive calls are only made to 2 randomly selected referenced peers.

\begin{tabular}{rrr}
\hline refmax & $e$ & $\frac{e}{N}$ \\
\hline \hline 1 & 23826 & 23.826 \\
2 & 37689 & 37.689 \\
3 & 40961 & 40.961 \\
4 & 43914 & 43.914 \\
\hline
\end{tabular}




\subsection{Search and Update Performance}

The subsequent simulations are based on a configuration that is similar to the one described in the example in Sec. 4. This confirms that the algorithms scale well for realistic parameter settings. We use 20000 peers that build a P-Grid with keys of maximal length 10 . The maximal number of references re fmax at each level is limited to 20 . The online probability of peers is $30 \%$.

Building a P-Grid of that size within a simulation environment requires considerable computing resources. Within approximately 10 hours of running time on a Pentium III processor the P-Grid was constructed up to an average depth of 9.43. During that time 1250743 exchanges among peers were performed, i.e. 62 per peer. Based on this P-Grid we make the following observations.

First we see that the replicas, i.e. the number of different peers responsible for the same key are fairly uniformly distributed. Figure 4 shows this result. The $\mathrm{x}$-axis indicates the replication factor and the $\mathrm{y}$-axis the number of peers that have this replication factor. The average number of replicas for a peer is 19.46.

A simple, intuitive argument shows that this is not surprising as the exchange function inherently tends to balance the distribution of keys. For example, a peer will decide upon the first bit of the key when it meets the first time another peer that has already taken this decision or also needs to decide on the first bit. It will decide in both cases opposite to the other peer. Thus, if there exists an imbalance in the distribution of the first bit, this is compensated for.

Next we would like to confirm the analysis of Sec. 4. We search 10000 times for a random key of length 9 . Only $30 \%$ of the peers are online. In $99.97 \%$ of the cases the search was successful and a search required on average 5.5576 messages among peers. We were counting as messages the successful calls of the query operation to another peer. This shows that searches can be performed reliably.

Now we turn to the question of how updates can be performed. The problem with an update, in contrast to a search, is, that we have to find all replicas of a path, not just one. Therefore we analyze how efficiently a large fraction of all replicas can be found. We compare the three approaches of (1) repeated depth

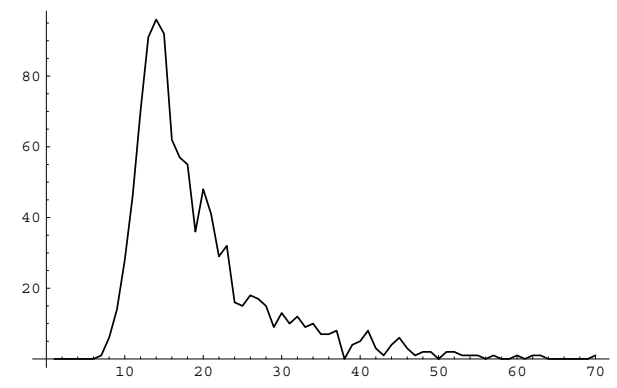

Fig. 4. Replica distribution 
first searches, (2) repeated depth first searches including all buddies that have been identified throughout index construction, and (3) repeated breadth first searches.

We repeatedly searched for a random key of length 9 and then computed the fraction of replicas identified as compared to the actual number of existing replicas. Figure 5 shows the result. The x-axis shows the number of messages used in the insertion process, and the y-axis the percentage of successfully identified replicas. One can see that clearly the strategy of using breadth first searches is by far superior, while the two other methods perform comparably.

One can see also, that for achieving a high update reliability a fairly large number of messages is required (hundreds per updated replica). So this approach is acceptable where updates are rare as compared to queries. However, the relevant problem is not to achieve high reliability in keeping the replicas consistent, but rather high reliability in obtaining correct query results. Thus we can use a different approach. We update a sufficiently high number of replicas using fewer messages, and use repeated query operations. Obviously, if more than half of the replicas are correct, by repeating queries, arbitrarily high reliability can be achieved by a making majority decision. In this manner we increase slightly the query cost and in turn reduce drastically the update cost. There is another factor that is helpful in that context. Not all replicas are as likely to be found. This implies that replicas that are found during updates are also more likely to be found during queries.

We perform a simulation to illustrate the tradeoff among update and query cost and indicate the optimal combinations of update and query strategies. 100 updates were performed and each updated data item was searched 10 times, thus 1000 queries were performed in each configuration. Updates are performed using breadth first search, where at each level recbreadth references are followed. The search was repeated repetition times. The successrate is the fraction of successfully answered queries after an update. The cost is in terms of number of messages.

The simulation shows that the approach of using repeated searches to achieve query reliability pays off dramatically. The configuration recbreadth $=3$ and

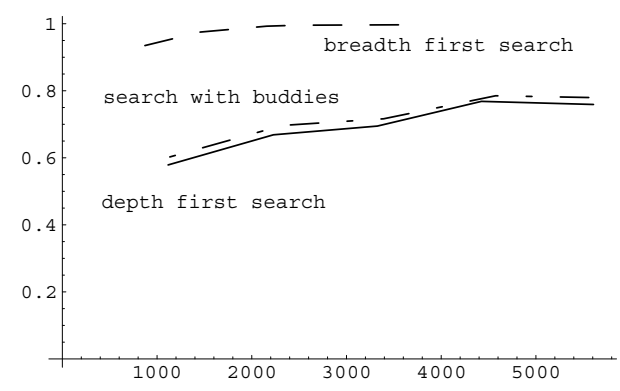

Fig. 5. Finding all replicas 
repetition $=3$ without using repeated search, which achieves only $99,4 \%$ reliability, would require at least a ratio of 160 queries per update in order reach the break-even point compared to the configuration recbreadth $=2$ and repetition $=$ 3 using repeated search, which offers practically $100 \%$ reliability.

\begin{tabular}{rrrrr}
\hline repetitive search & \multicolumn{3}{c}{} \\
\hline \hline recbreadth & repetition & successrate & query cost & insertion cost \\
2 & 1 & 1 & 137 & 78 \\
2 & 2 & 1 & 34 & 147 \\
2 & 3 & 1 & 17 & 224 \\
3 & 1 & 1 & 112 & 637 \\
3 & 2 & 1 & 13 & 1434 \\
3 & 3 & 1 & 13 & 2086 \\
\hline non-repetitive search & & & & \\
\hline \hline 2 & 1 & 0.65 & 5.5 & 145 \\
2 & 2 & 0.85 & 5.6 & 212 \\
2 & 3 & 0.89 & 5.4 & 734 \\
3 & 1 & 0.95 & 5.5 & 1363 \\
3 & 2 & 0.98 & 5.5 & 2080 \\
\hline 3 & 3 & 0.994 & 5.4 &
\end{tabular}

\section{Discussion}

This paper introduced P-Grid, a first step towards developing scalable, robust and self-organizing access structures for P2P information systems. To illustrate the effectiveness of a P-Grid we can compare it to centralized replicated server architectures. Assume $D$ is the number of data items and $N$ the number of peers. For storage we consider the number of references to be stored at the nodes ignoring local indexing cost. For querying we consider the number of messages exchanged assuming that each node creates a constant number of queries per time unit. Then a solution using centralized replicated servers compares to PGrid as follows.

\begin{tabular}{lll}
\hline & P-Grid & Central Server \\
\hline \hline Storage & peers: $O(\log D)$ & $\begin{array}{l}\text { server: } O(D) \\
\text { client: constant } \\
\text { Query }\end{array}$ \\
& peers: $O(\log N)$ & $\begin{array}{l}\text { server: } O(N) \\
\text { client: constant }\end{array}$ \\
\hline
\end{tabular}

One can see that both storage and communication cost scale well for the PGrid. For a centralized servers the linear growth of communication cost in the client number is critical as servers become bottlenecks. This shows that besides robustness also scalability is an asset of P-Grids.

The approach presented in this paper is limited to uniform data distributions. For uniformly distributed key values the P-Grid can be immediately applied. For prefix search on text the algorithm can be adapted by extending the $\{0,1\}$ alphabet. This would allow to directly support trie search structures. However 
the decentralized support for more sophisticated search structures, like in [3] is a challenging research topic.

An obvious continuation of this research is to develop P-Grids that can support skewed data distributions. To that extent throughout the construction process the actual data distribution needs to be taken into account and the structures have to continuously adapt to updates. Another natural extension of the approach would be to take system parameters, like known reliability of peers, knowledge on the network topology or knowledge on query distribution into account for optimizing P-Grid construction and updates. To achieve load balancing a computational economy can be imposed, as already investigated for distributed data mangement in [4][9].

We see the P-Grid, as it is presented in this paper, as a first representative of access structures for $\mathrm{P} 2 \mathrm{P}$ information systems, for which many variations will be developed, that are adapted to the specific requirements of various $\mathrm{P} 2 \mathrm{P}$ application domains.

Acknowledgements. I would like to thank Manfred Hauswirth and Roman Schmidt for carefully reading and commenting the manuscript. I would also like to thank Magdalena Punceva and Rachid Guerraoui for many helpful discussions. This work also greatly benefited from the inspiring working environment that is provided by the newly founded Communication Systems Department at EPFL.

\section{References}

1. E. Adar and B. A. Huberman: Free riding on Gnutella Technical report, Xerox PARC, 10 Aug. 2000.

2. D. Clark. Face-to-Face with Peer-to-Peer Networking. IEEE Computer, January 2001.

3. Y. Chen, K. Aberer: Combining Pat-Trees and Signature Files for Query Evaluation in Document Databases DEXA 99, LNCS, Vol. 1677, p. 473-484, Springer, 1999.

4. D.F. Ferguson, C. Nikolaou and Y. Yemini An Economy for Managing Replicated Data in Autonomous Decentralized Systems International Symposium on Autonomous Decentralized Systems (ISADS'93), 1993.

5. T. Johnson, P. Krishna Lazy Updates for Distributed Search Structure ACM SIGMOD 93, p. 337-346, 1993.

6. B. Kröll, P. Widmayer Distributing a Search Tree Among a Growing Number of Processors. ACM SIGMOD 94, p. 265-276, 1994.

7. B. Kröll, P. Widmayer Balanced Distributed Search Trees Do Not Exist WADS 95, p 50-61, 1995.

8. W. Litwin, M. Neimat, D. A. Schneider $R P^{*}$ : A Family of Order Preserving Scalable Distributed Data Structures. VLDB 94, p. 342-353, 1994.

9. M. Stonebraker, P. M. Aoki, W. Litwin, A. Pfeffer, A. Sah, Jeff Sidell, Carl Staelin, Andrew Yu: Mariposa A Wide-Area Distributed Database System VLDB Journal 5(1): 48-63, 1996.

10. R. Vingralek, Y. Breitbart, G. Weikum SNOWBALL: Scalable Storage on Networks of Workstations with Balanced Load Distributed and Parallel Databases Vol 6(2), Kluwer Academic Publishers, 1998.

11. H. Yokota, Y. Kanemasa, J. Miyazaki Fat-Btree: An Update-Conscious Parallel Directory Structure ICDE 99, p. 448-457, 1999. 